http://journal.uinsgd.ac.id/index.php/biodjati

\title{
Dinochloa scandens (POACEAE-BAMBUSOIDEAE): DISTRIBUTION, HABITAT PREFERENCE, AND NOTES ON SYNONYMY
}

\section{Putu Gede P. Damayanto ${ }^{1 *}$, Syadwina H. Dalimunthe², Megawati $^{3}$}

Received : May 12, 2021

Accepted : August 10, 2021

DOI: 10.15575/biodjati.v6i2.12485

${ }^{1,2,3}$ Herbarium Bogoriense, Research Center for Biology, Indonesian Institute of Sciences (LIPI),

Jl. Raya Jakarta-Bogor Km 46, Cibinong, Bogor, West Java, 16911, Indonesia

e-mail:

*Iparlida.damayanto.tab@gmail.com

hamamabie@gmail.com

3mgdhuqun@gmail.com

*Corresponding author

Abstract. The distribution of Dinochloa scandens is unclear. World bamboo checklists suggest it is relatively widespread in Malesia. Here we clarify issues with herbarium specimen identification. Besides, a synonym name of D. scandens, namely D. macrocarpa collected from the Philippines, needs to be reviewed. The study aims to provide information on distribution and to review the synonym of $D$. scandens. The habitat preferences of D. scandens are also presented. We carried out targeted fieldwork at Gunung Halimun-Salak National Park in West Java in 2019 and extensive examination of herbarium specimens from Herbarium Bogoriense and online portals to accurately circumscribe this species. All data were analyzed descriptively. Distribution, altitude, and rainfall maps for D. scandens were produced with ArcGIS Pro. Our examination on the specimens suggests that D. scandens is indeed endemic to West Java and Banten Provinces in Indonesia. This bamboo species can be found in lowland to mountain forests with an altitude of 20-1400 m, in areas with annual rainfall between 3000-4000 $\mathrm{mm}$. We support D. macrocarpa as an accepted name for a bamboo species from the Philippines and remove it from synonymy with $D$. scandens due to clear differences in the fruit compared to specimens of D. scandens across its range.

Keywords: bamboo, Dinochloa macrocarpa, Dinochloa scandens, distribution, habitat preference

\section{Citation}

Damayanto, I. P. G. P., Dalimunthe, S. H. \& Megawati. (2021). Dinochloa scandens (Poaceae-Bambusoideae): Distribution, Habitat Preference, and Notes on Synonymy. Jurnal Biodjati, 6(2), 174-189.

\section{INTRODUCTION}

Dinochloa

Buse

(PoaceaeBambusoideae) is a genus of wild climbing bamboo found mainly in Malesia but also the Andaman Islands and Southern Thailand. Most Dinochloa species have a narrow distribution and are commonly found in disturbed forests. Dinochloa is easily identified by its climbing habit, solid culms, very small clusters of spikelets, and comparatively large fruit (Dransfield, 1981). The climbing habit in Dinochloa is very different from the habit of other bamboos. The culms twine to the neighbor tree trunk (always twining to the right), however, the culms become zigzagged if there is no support for climbing. The lower part of the older culmsheath of Dinochloa is very rough and thick (the term "girdle" is often used), this is not visible on young culm-sheaths. The climbing habit and "girdle" feature on Dinochloa 


\section{JURNAL BIDDJATI}

http://journal.uinsgd.ac.id/index.php/biodjati

are very useful to identify the genus.

Dinochloa was published in 1854 by Buse, the type species being D. tjankorreh Buse (see Buse, 1854). Nowadays, the accepted name of $D$. tjankorreh is $D$. scandens (Blume ex Nees) Kuntze (see Kuntze, 1891; Dransfield, 1994; Dransfield \& Widjaja, 1995; Vorontsova et al., 2016). At first glance, a sterile $D$. scandens can be identified by its purplish and smooth young shoot, culm with solid or with small lumen up to $15 \mathrm{~mm}$ in diameter, internodes rather rough with appressed hairs when young and becoming smooth and glabrous when mature. Culmsheaths are glabrous or sometimes have white hairs, a very small or inconspicuous auricle, and a very short ligule

Dransfield (1994, 1996), Dransfield \& Widjaja (1995), Damayanto (2018), and Widjaja (2019) mentioned that D. scandens has a limited distribution area in Java. However, Vorontsova et al. (2016), a database of Plant of the World (POWO) website (http:// powo.science.kew.org/taxon/urn:1sid:ipni. org:names:399398-1), the Global Biodiversity Information Facility (GBIF) (https://www. gbif.org/species/4149851) and some studies (Priyadi et al., 2010; Cleary, 2016; Jamun et al., 2020; Sharief \& Panda, 2020; Sujarwanta $\&$ Zen, 2020a, 2020b) suggest that $D$. scandens can be found outside of Java (e.g. Sumatra, Borneo, Lesser Sunda Islands, the Andaman Islands, Nicobar Islands, and Thailand). Whether D. scandens is a Java endemic or more widespread needs to be confirmed.

In addition, D. macrocarpa Elmer collected from the type locality at Magallanes (Mt. Giting-giting) (the correct name is Mt. Guiting-Guiting), Sibuyan Islands, Province of Capiz (now Romblon Province), in the Philippines (Elmer, 1915) was reported as a synonym of $D$. scandens (Vorontsova et al., 2016). This contradicted the statements of
Dransfield (1994, 1996), Dransfield \& Widjaja (1995), Damayanto (2018), and Widjaja (2019), who stated that D. scandens was endemic to western Java. A study, therefore, is needed to clarify the distribution and synonyms of $D$. scandens. We also explore the habitat preferences (annual rainfall and topography) of $D$. scandens. The results hopefully can be useful in habitat conservation of $D$. scandens.

\section{MATERIALS AND METHODS}

This study consisted of herbarium specimen examination and targeted fieldwork. The herbarium specimens of D. scandens deposited at Herbarium Bogoriense (BO), Research Center for Biology, Indonesian Institute of Sciences (LIPI) and online portals were examined. Coordinates were compiled from the data of $\mathrm{BO}$ specimens and other digital specimens that were available at portal online databases such as BioPortal (https://bioportal.naturalis.nl/), JSTOR (https://plants.jstor.org/) GBIF (https://www. gbif.org/), HerbWeb (https://apps.kew.org/ herbcat/gotoHomePage.do), iDigBio (https:// www.idigbio.org/portal/search), POWO (http://powo.science.kew.org/) and Tropicos (https://www.tropicos.org/home). Collection localities lacking coordinates were matched to locations in the Encyclopaedia of Malesian Collectors (Steenis-Kruseman, 1950) or the website http://nationaalherbarium. nl/FMCollectors/QuickSearch.htm and coordinates gathered using Google Maps (https://www.google.com/maps). The initial status of accepted species names followed Vorontsova et al. (2016) or websites such as IPNI (ipni.org) and POWO (powo.science. kew.org). Code of the herbarium followed website http://sweetgum.nybg.org/science/ $\mathrm{ih} /$ or Girmansyah et al. $(2006,2018)$ and Holmgren et al. (1981). Furthermore, the type 


\section{JURNAL BIODJATI}

http://journal.uinsgd.ac.id/index.php/biodjati

specimen of D. macrocarpa was examined and compared with specimens of $D$. scandens.

Targeted plant collection took place in October 2019, at Cikaniki Resort Station, Gunung Halimun-Salak National Park, West Java, Indonesia. Bamboo samples were collected following McClure (1945) and processed as herbarium specimens following Djarwaningsih et al. (2002). Supporting data, such as coordinates, altitude, habitat, local name, uses, were recorded and photographs of field specimens were taken. Samples were processed and stored at BO. Furthermore, study of the living collection of $D$. scandens was also conducted. Bamboo materials were identified by comparing with herbarium specimens stored in BO and using literature such as Dransfield (1981, 1989, 1992, 1996), Widjaja (1997, 2001a, 2001b, 2009), Widjaja et al. (2004), Dransfield \& Widjaja (2000), and Ervianti et al. (2019).

Dinochloa scandens distribution maps across elevation and annual rainfall gradients were plotted using ArcGIS Pro software. Habitat preference was taken as the range of environmental variables covered by the distribution of specimens.

\section{RESULTS AND DISCUSSION}

Ninety-four sheets of 35 herbarium specimens, as well as one living collection of $D$. scandens, were examined. These specimens were derived from fieldwork and those already stored in Herbarium Bogoriense (BO), Herbarium Royal Botanic Gardens, Kew (K), and National Herbarium Nederland (L), and living plant collections in Bogor (Table 1). Most of the sheets of $D$. scandens were observed from BO collections (84 sheets or $89 \%$ ) and the others from L (9 sheets or $10 \%$ ) and $\mathrm{K}$ ( 1 sheet or $1 \%)$. The oldest collection of $D$. scandens was from 1899 and the newest 2019 (Figure 1). Based on Figure 1, only one new collection was added in BO after 2000 (a collection from 2019 fieldwork).

Table 1. Observation matrix of herbarium specimens and living collection of Dinochloa scandens

\begin{tabular}{|c|c|c|c|c|c|c|c|}
\hline \multirow[t]{2}{*}{ Collector } & \multirow{2}{*}{$\begin{array}{l}\text { Cellection } \\
\text { Number }\end{array}$} & \multirow{2}{*}{$\begin{array}{l}\text { Collection } \\
\text { Date }\end{array}$} & \multicolumn{2}{|c|}{\begin{tabular}{l|l} 
Location & \\
\end{tabular}} & \multirow{2}{*}{$\begin{array}{l}\text { Coordinates } \\
\text { Prediction }\end{array}$} & \multirow{2}{*}{$\begin{array}{c}\text { Altitude } \\
\text { (m asl) }\end{array}$} & \multirow{2}{*}{$\begin{array}{l}\text { Herbaria } \\
\text { Code }\end{array}$} \\
\hline & & & $\begin{array}{l}\text { Recorded on the } \\
\text { Specimen }\end{array}$ & $\begin{array}{l}\text { Current } \\
\text { Location }\end{array}$ & & & \\
\hline $\begin{array}{l}\text { C. A. } \\
\text { Backer }\end{array}$ & 1292 & $\begin{array}{l}\text { 13 Jun. } \\
1911\end{array}$ & $\begin{array}{l}\text { Java, Bantam, } \\
\text { G. Kencana, G. } \\
\text { Kendeng }\end{array}$ & $\begin{array}{l}\text { Banten, Lebak, } \\
\text { Gunungkencana }\end{array}$ & $\begin{array}{l}6^{\circ} 33^{\prime} 32.4^{\prime \prime} \mathrm{S}, \\
106^{\circ} 03^{\prime} 47.2^{\prime \prime} \mathrm{E}\end{array}$ & 300 & $\mathrm{BO}$ \\
\hline $\begin{array}{l}\text { C. A. } \\
\text { Backer }\end{array}$ & 8731 & $\begin{array}{l}17 \text { Aug. } \\
1913\end{array}$ & $\begin{array}{l}\text { Java, Preanger } \\
\text { Regentschappen, } \\
\text { G. Tjikoraj, Z. O. } \\
\text { Helling, Pasir Walang } \\
\text { Boven Nanggerang }\end{array}$ & $\begin{array}{l}\text { West Java, } \\
\text { Garut, Mt. } \\
\text { Cikuray }\end{array}$ & $\begin{array}{l}7^{\circ} 19^{\prime} 29.6 ” S, \\
107^{\circ} 51 ' 31.7^{\prime} \mathrm{E}\end{array}$ & $\begin{array}{l}950- \\
1000\end{array}$ & $\mathrm{BO}$ \\
\hline $\begin{array}{l}\text { C. A. } \\
\text { Backer }\end{array}$ & 8731 & 1913 & $\begin{array}{l}\text { Java, Pasir Walang, } \\
\text { Nanggerang }\end{array}$ & $\begin{array}{l}\text { Banten, Lebak, } \\
\text { Pasir Walang }\end{array}$ & $\begin{array}{l}6^{\circ} 50^{\prime} 09.0^{\prime \prime} \mathrm{S}, \\
106^{\circ} 13 \text { '34.1”E }\end{array}$ & - & $\mathrm{BO}$ \\
\hline $\begin{array}{l}\text { C. A. } \\
\text { Backer }\end{array}$ & 11068 & $\begin{array}{l}24 \text { Dec. } \\
1913\end{array}$ & $\begin{array}{l}\text { Java, Batavia, } \\
\text { Nirmala }\end{array}$ & $\begin{array}{l}\text { West Java, } \\
\text { Bogor, } \\
\text { Nanggung, } \\
\text { Malasari } \\
\text { Village, } \\
\text { Nirmala }\end{array}$ & $\begin{array}{l}6^{\circ} 44^{\prime} 00.8^{\prime \prime} \mathrm{S}, \\
106^{\circ} 29^{\prime} 59.9^{\prime} \mathrm{E}\end{array}$ & 1100 & $\mathrm{BO}$ \\
\hline $\begin{array}{l}\text { C. A. } \\
\text { Backer }\end{array}$ & $22941 *$ & $\begin{array}{l}09 \text { Sep. } \\
1917\end{array}$ & $\begin{array}{l}\text { Java, Preanger } \\
\text { Regentschappen, } \\
\text { Tjoeroeg Djanggot, } \\
\text { Cidadap, Cibeber }\end{array}$ & $\begin{array}{l}\text { West Java, } \\
\text { Cianjur, } \\
\text { Cibeber }\end{array}$ & $\begin{array}{l}6^{\circ} 55^{\prime} 37.5^{\prime \prime} \mathrm{S}, \\
107^{\circ} 08^{\prime} 55.7^{\prime} \mathrm{E}\end{array}$ & 900 & $\mathrm{BO}$ \\
\hline
\end{tabular}




\section{JURNAL BIDDJATI}

http://journal.uinsgd.ac.id/index.php/biodjati

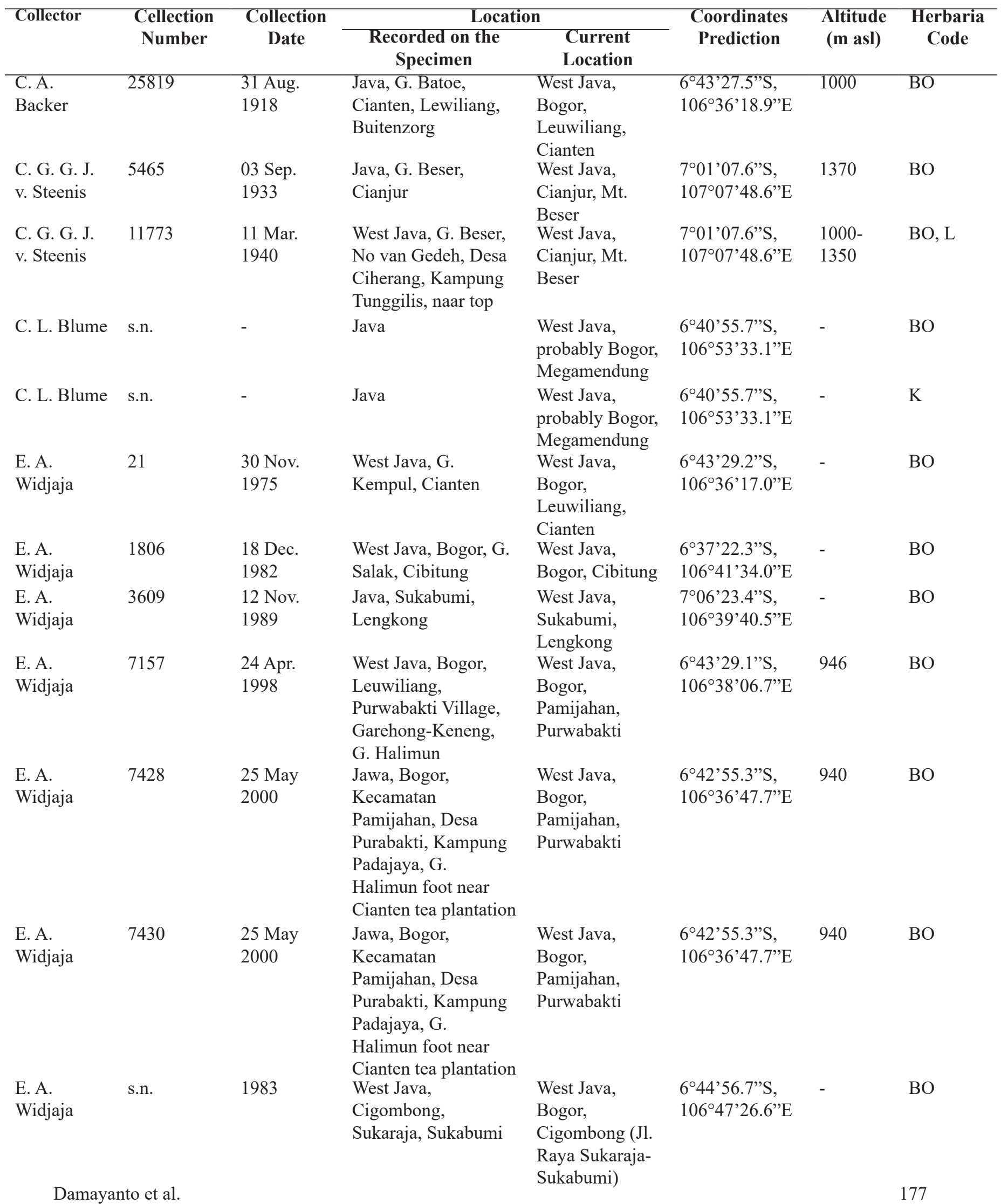




\section{JURNAL BIDDJATI}

http://journal.uinsgd.ac.id/index.php/biodjati

\begin{tabular}{|c|c|c|c|c|c|c|c|}
\hline \multirow[t]{2}{*}{ Collector } & \multirow{2}{*}{$\begin{array}{l}\text { Cellection } \\
\text { Number }\end{array}$} & \multirow{2}{*}{$\begin{array}{l}\text { Collection } \\
\text { Date }\end{array}$} & \multicolumn{2}{|c|}{\begin{tabular}{l|l} 
Location \\
\end{tabular}} & \multirow{2}{*}{$\begin{array}{l}\text { Coordinates } \\
\text { Prediction }\end{array}$} & \multirow{2}{*}{$\begin{array}{l}\text { Altitude } \\
\text { (m asl) }\end{array}$} & \multirow{2}{*}{$\begin{array}{l}\text { Herbaria } \\
\text { Code }\end{array}$} \\
\hline & & & $\begin{array}{l}\text { Recorded on the } \\
\text { Specimen }\end{array}$ & $\begin{array}{l}\text { Current } \\
\text { Location }\end{array}$ & & & \\
\hline $\begin{array}{l}\text { G. G. } \\
\text { Hambali }\end{array}$ & 722 & $\begin{array}{l}\text { 15 Jun. } \\
1975\end{array}$ & $\begin{array}{l}\text { West Java, Ga. } \\
\text { Malang, Takoka Nat. } \\
\text { Res. }\end{array}$ & $\begin{array}{l}\text { West Java, } \\
\text { Cianjur, } \\
\text { Takokak }\end{array}$ & $\begin{array}{l}7^{\circ} 07^{\circ} 47.8^{\prime \prime} \mathrm{S}, \\
107^{\circ} 00^{\prime} 03.1{ }^{\prime \prime} \mathrm{E}\end{array}$ & 1100 & BO \\
\hline $\begin{array}{l}\text { H. } \\
\text { Wiriadinata } \\
\text { \& W. S. } \\
\text { Hoover }\end{array}$ & 31193 & $\begin{array}{l}22 \text { Feb. } \\
2000\end{array}$ & $\begin{array}{l}\text { West Java, Mount } \\
\text { Salak, Facing East, } \\
\text { Upper Lido Village }\end{array}$ & $\begin{array}{l}\text { West Java, } \\
\text { Bogor, } \\
\text { Cigombong }\end{array}$ & $\begin{array}{l}6^{\circ} 43^{\prime} 32.8^{\prime \prime} \mathrm{S}, \\
106^{\circ} 46^{\prime} 40.8^{\prime} \mathrm{E}\end{array}$ & $\begin{array}{l}1000- \\
1200\end{array}$ & $\mathrm{BO}$ \\
\hline $\begin{array}{l}\text { H. } \\
\text { Wiriadinata, } \\
\text { W. S. Hoover } \\
\text { \& J. Hunter }\end{array}$ & 31339 & 07 Mar. 2000 & $\begin{array}{l}\text { West Java, Mt. Halimun } \\
\text { Nat. Park Cikaniki }\end{array}$ & $\begin{array}{l}\text { West Java, Mt. } \\
\text { Halimun National } \\
\text { Park, Cikaniki }\end{array}$ & $\begin{array}{l}6^{\circ} 44^{\prime} 47.5^{\prime \prime} \mathrm{S} \\
106^{\circ} 32^{\prime} 13.7^{\prime \prime} \mathrm{E}\end{array}$ & $\begin{array}{l}1000- \\
1400\end{array}$ & $\mathrm{BO}$ \\
\hline Hallier & s.n. & 02 May 1933 & $\begin{array}{l}\text { Java, Mount Salak, } \\
\text { Prope Tjoeroeg }\end{array}$ & $\begin{array}{l}\text { West Java, Bogor, } \\
\text { Mt. Salak }\end{array}$ & $\begin{array}{l}6^{\circ} 42^{\prime} 46.1 " \mathrm{~S} \\
106^{\circ} 44^{\prime} 34.4 \text { 'E }\end{array}$ & - & $\mathrm{BO}$ \\
\hline $\begin{array}{l}\text { J. v. B. } \\
\text { Waalkes }\end{array}$ & 468 & 19 Sep. 1951 & $\begin{array}{l}\text { Java, Pulau Panaitan } \\
\text { (Prinseneiland) between } \\
\text { Tg. Manik and N. Coast } \\
\text { (W. Peninsula) }\end{array}$ & $\begin{array}{l}\text { Banten, } \\
\text { Pandeglang, } \\
\text { Panaitan Island }\end{array}$ & $\begin{array}{l}6^{\circ} 35^{\prime} 20.2^{\prime \prime} \mathrm{S} \\
105^{\circ} 12^{\prime} 24.3^{\prime \prime} \mathrm{E}\end{array}$ & 20 & $\mathrm{BO}, \mathrm{L}$ \\
\hline leg. ign. & s.n. & - & Java, Mount Salak & $\begin{array}{l}\text { West Java, Mt. } \\
\text { Salak }\end{array}$ & $\begin{array}{l}6^{\circ} 43 \text { '09.5”S, } \\
106^{\circ} 44^{\prime} 13.1 \text { 'E }\end{array}$ & - & $\mathrm{BO}$ \\
\hline leg. ign. & s.n. & - & $\begin{array}{l}\text { Java, Mount. Salak, } \\
\text { Prope Tjoeroeg }\end{array}$ & $\begin{array}{l}\text { West Java, Mt. } \\
\text { Salak }\end{array}$ & $\begin{array}{l}6^{\circ} 42^{\prime} 48.9 \text { 'S, } \\
106^{\circ} 44^{\prime} 09.9 \text { 'Е }\end{array}$ & - & $\mathrm{BO}$ \\
\hline N. Wirawan & s.n. & 31 Dec. 1963 & $\begin{array}{l}\text { S. W. Java, Ujungkulon } \\
\text { Nature Reserve, } \\
\text { Gunung Pajung }\end{array}$ & $\begin{array}{l}\text { Banten, } \\
\text { Pandeglang, } \\
\text { Ujungkulon } \\
\text { Nature Reserve }\end{array}$ & $\begin{array}{l}6^{\circ} 49^{\prime} 04.4 ” \mathrm{~S}, \\
105^{\circ} 15^{\prime} 59.5^{\prime} \mathrm{E}\end{array}$ & 400 & $\mathrm{BO}$ \\
\hline $\begin{array}{l}\text { R. C. } \\
\text { Bakhuizen } \\
\text { v/d Brink }\end{array}$ & 1617 & 12 Jun. 1916 & $\begin{array}{l}\text { Java, Preanger, } \\
\text { Cidadap, Cibeber }\end{array}$ & $\begin{array}{l}\text { West Java, } \\
\text { Cianjur, Cibeber }\end{array}$ & $\begin{array}{l}6^{\circ} 55^{\prime} 13.0 ” \mathrm{~S} \\
107^{\circ} 05^{\prime} 20.7^{\prime \prime} \mathrm{E}\end{array}$ & 1000 & $\mathrm{BO}, \mathrm{L}$ \\
\hline $\begin{array}{l}\text { R. C. } \\
\text { Bakhuizen }\end{array}$ & 4393 & 23 Jul. 1920 & $\begin{array}{l}\text { Java, Res. Batavia, G. } \\
\text { Limoes (Boerangrang) }\end{array}$ & $\begin{array}{l}\text { West Java, } \\
\text { Burangrang }\end{array}$ & $\begin{array}{l}6^{\circ} 46^{\prime} 19.4 ” S, \\
107^{\circ} 34^{\prime} 17.0 ” \mathrm{E}\end{array}$ & 1200 & $\mathrm{BO}$ \\
\hline $\begin{array}{l}\text { v/d Brink } \\
\text { S. H. } \\
\text { Dalimunthe }\end{array}$ & 20 & 9 Oct. 2019 & $\begin{array}{l}\text { West Java, Cikaniki } \\
\text { Resort Station }\end{array}$ & $\begin{array}{l}\text { West Java, } \\
\text { Cikaniki Resort } \\
\text { Station }\end{array}$ & $\begin{array}{l}6^{\circ} 44^{\prime} 47.2 ” S, \\
106^{\circ} 32 ’ 16.2 ” \mathrm{E}\end{array}$ & $\begin{array}{l}1000- \\
1200\end{array}$ & $\mathrm{BO}$ \\
\hline $\begin{array}{l}\text { S. H. } \\
\text { Koorders }\end{array}$ & 15093 & 16 Feb. 1984 & $\begin{array}{l}\text { Java, Preanger, } \\
\text { Boschterrein Takoka, } \\
\text { Distr. Djampang-Wetan, } \\
\text { Afd. Tjiandjoer }\end{array}$ & $\begin{array}{l}\text { West Java, } \\
\text { Cianjur (Jampang } \\
\text { Wetan) }\end{array}$ & $\begin{array}{l}6^{\circ} 48^{\prime} 07.3 ” S \\
107^{\circ} 08^{\prime} 30.6 \text { 'E }\end{array}$ & 1000 & $\mathrm{BO}, \mathrm{L}$ \\
\hline $\begin{array}{l}\text { S. H. } \\
\text { Koorders }\end{array}$ & 32907 & 07 Apr. 1899 & $\begin{array}{l}\text { Java, Tjiandjoer / } \\
\text { Preanger, Tokoka }\end{array}$ & $\begin{array}{l}\text { West Java, } \\
\text { Cianjur }\end{array}$ & $\begin{array}{l}6^{\circ} 48^{\prime} 54.1 " \mathrm{~S} \\
107^{\circ} 08^{\prime} 07.3 \text { ” E }\end{array}$ & 1000 & $\mathrm{BO}$ \\
\hline $\begin{array}{l}\text { S. H. } \\
\text { Koorders }\end{array}$ & 41410 & 09 Jun. 1912 & $\begin{array}{l}\text { Java, Goenoeng } \\
\text { Kantjang, Distr. Lebak } \\
\text { Kidoel, Res. Bantam }\end{array}$ & $\begin{array}{l}\text { Banten, Lebak, } \\
\text { Gunungkencana }\end{array}$ & $\begin{array}{l}6^{\circ} 34^{\prime} 43.0^{\prime \prime} \mathrm{S}, \\
106^{\circ} 01^{\prime} 59.4 \text { 'E }\end{array}$ & - & $\mathrm{BO}$ \\
\hline $\begin{array}{l}\text { W. F. } \\
\text { Winckel }\end{array}$ & 220 & 03 Jul. 1918 & $\begin{array}{l}\text { Java, Preanger, G. } \\
\text { Beser, Tjidadap, } \\
\text { Tjibeber }\end{array}$ & $\begin{array}{l}\text { West Java, } \\
\text { Cianjur, Cibeber }\end{array}$ & $\begin{array}{l}6^{\circ} 56^{\prime} 26.6^{\prime \prime} \mathrm{S} \\
107^{\circ} 08^{\prime} 06.4^{\prime \prime} \mathrm{E}\end{array}$ & 1000 & $\mathrm{BO}, \mathrm{L}$ \\
\hline $\begin{array}{l}\text { W. F. } \\
\text { Winckel }\end{array}$ & 449 & 26 Oct. 1919 & $\begin{array}{l}\text { Java, Preanger, Tjadas } \\
\text { Malang, Cibeber }\end{array}$ & $\begin{array}{l}\text { West Java, } \\
\text { Cianjur, Cadas }\end{array}$ & $\begin{array}{l}7^{\circ} 03^{\prime} 01.0 ’ \mathrm{~S} \\
107^{\circ} 10^{\prime} 01.0^{\prime \prime} \mathrm{E}\end{array}$ & 1000 & $\mathrm{BO}, \mathrm{L}$ \\
\hline $\begin{array}{l}\text { W. F. } \\
\text { Winckel }\end{array}$ & 1741 & 20 Oct. 1923 & $\begin{array}{l}\text { Java, Preanger, } \\
\text { Goenoeng Boeled, } \\
\text { Cidadap, Cibeber }\end{array}$ & $\begin{array}{l}\text { Malang } \\
\text { West Java, } \\
\text { Cianjur, Mt. } \\
\text { Buleud }\end{array}$ & $\begin{array}{l}7^{\circ} 05^{\prime} 48.4^{\prime \prime} \mathrm{S}, \\
107^{\circ} 03^{\prime} 15.0^{\prime} \mathrm{E}\end{array}$ & 1000 & $\mathrm{BO}, \mathrm{L}$ \\
\hline
\end{tabular}




\section{JURNAL BIDDJATI}

http://journal.uinsgd.ac.id/index.php/biodjati

\begin{tabular}{|c|c|c|c|c|c|c|c|}
\hline \multirow[t]{2}{*}{ Collector } & \multirow{2}{*}{$\begin{array}{l}\text { Cellection } \\
\text { Number }\end{array}$} & \multirow{2}{*}{$\begin{array}{l}\text { Collection } \\
\text { Date }\end{array}$} & \multicolumn{2}{|c|}{\begin{tabular}{r|r|} 
Location \\
\end{tabular}} & \multirow{2}{*}{$\begin{array}{l}\text { Coordinates } \\
\text { Prediction }\end{array}$} & \multirow{2}{*}{$\begin{array}{l}\text { Altitude } \\
\text { (m asl) }\end{array}$} & \multirow{2}{*}{$\begin{array}{l}\text { Herbaria } \\
\text { Code }\end{array}$} \\
\hline & & & $\begin{array}{l}\text { Recorded on the } \\
\text { Specimen }\end{array}$ & $\begin{array}{l}\text { Current } \\
\text { Location }\end{array}$ & & & \\
\hline W. Meijer & 2949 & 20 Aug. 1954 & $\begin{array}{l}\text { West Java, W. of } \\
\text { Djasinga, Forest- } \\
\text { Reserve Djanglope }\end{array}$ & $\begin{array}{l}\text { West Java, Bogor, } \\
\text { West Part of } \\
\text { Jasinga }\end{array}$ & $\begin{array}{l}6^{\circ} 28^{\prime} 50.4^{\prime \prime} \mathrm{S}, \\
106^{\circ} 24^{\prime} 56.4^{\prime} \mathrm{E}\end{array}$ & 900 & $\mathrm{BO}$ \\
\hline $\begin{array}{l}\text { Living } \\
\text { collection }\end{array}$ & - & - & - & $\begin{array}{l}\text { West Java, Bogor, } \\
\text { Bogor Botanic } \\
\text { Gardens. LIPI }\end{array}$ & $\begin{array}{l}6^{\circ} 36^{\prime} 00.0^{\prime \prime} \mathrm{S}, \\
106^{\circ} 47^{\prime} 44.0^{\prime \prime} \mathrm{E}\end{array}$ & 270 & - \\
\hline
\end{tabular}

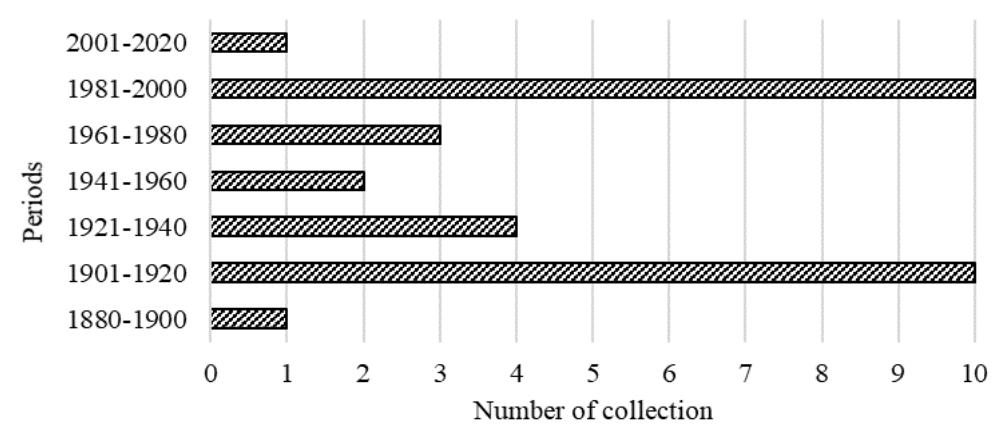

Figure 1. Number of herbarium specimens of Dinochloa scandens during twenty years time periods

\section{Taxonomic Account}

Dinochloa scandens (Blume ex Nees) Kuntze, Revis. Gen. P1. 2: 773 (1891). Type: Java, Megamendung, Blume s.n. (L holotype).

Synonyms: Bambusa scandens Blume ex Nees in Flora 7: 291 (1824) (basionym); Nastus tjankorreh Schult.f., Syst. Veg. 7(2): 1358 (1830) (nom. superfl. or superfluous name); Dinochloa tjankorreh Buse in Miquel, Pl. Jungh., prepr.: 388 (1854) (comb. superfl. or superfluous combination name); Chusquea amplopaniculata Steud., Syn. Pl. Glumac. 1(3): 337 (1854) (a synonym by Clark, 1986); Schizostachyum parviflorum Munro, Trans. Linn. Soc. London 26(1): 153 (1868) (a synonym by Munro, 1868).

Description (Figure 2-3): Climbing or twining with zig-zag culm. Young shoots purplish, glabrous, or occasionally with white wax, blade erects first then deflexed and easily fall. Culms purplish-green when young becoming green with age, solid or with small lumen, 9-20 $\mathrm{mm}$ diameters, internodes $17-24 \mathrm{~cm}$ long and slightly rough when young becoming glabrous. Branch complements consist of a primary branch and several secondary branches. The primary branch becomes dormant and develops into a full-size culm when the apex of the main culm is broken. Culm-sheaths caducous, usually glabrous or sometimes covered with pale hairs, 8-14 $\mathrm{cm}$ long and 4-5 $\mathrm{cm}$ wide near the base, narrower at the junction with the blade; the lower part of the culm-sheath (girdle) rough and thick; auricles inconspicuous up to $1 \mathrm{~mm}$ high, glabrous or rarely with bristles; ligule entire, 1-1.5 mm long; blades easily fall, erect first then deflexed, 5-8 cm long, wide near the base. Leaf-blades purplish-green when young becoming green with age, $11-19.5 \mathrm{~cm} \times 1.3-4$ $\mathrm{cm}$, glabrous; auricles small, less than $1 \mathrm{~mm}$ high, with bristles 2-5 mm long; ligule entire up to $1 \mathrm{~mm}$ high. Inflorescences are 120-50 $\mathrm{cm}$ long. Spikelets 3.5-4 mm long. Fruits are not available but Dransfield (1996) mentioned about $7 \mathrm{~mm}$ long, globose, and smooth. Vernacular names and uses: The vernacular name of this species is awi 


\section{JURNAL BIDDJATI}

http://journal.uinsgd.ac.id/index.php/biodjati

cangkoreh, cangkoreh or cangkore (Sundanese). The local people often use this bamboo as rope when there are no other plants that can be used in the forest (Widjaja, 2001b). The culms are also used for making handicrafts and rough baskets to carry stones from the rivers. The water of the culm of this bamboo is traditionally used as cough medicine, eye drops (Partasasmita et al., 2017; Setiawati et al., 2017), treat conjunctivitis, and anti-inflammatory (Priyadi et al., 2010). In Mt. Halimun, West Java, D. scandens is known as a host of leafhoppers Cofana yukawai Kamitani, 2004 (Hemiptera: Auchenorrhyncha: Cicadellidae) (Kamitani et al., 2004).

Notes:HolotypespecimenofD. scandens is collected from Megamendung, JavabyBlume (without number collection) and deposited in L (Dransfield, 1996). Unfortunately, this holotype was not available in portal databases of Herbarium $L$ that can be accessed at BioPortal (https://bioportal.naturalis.nl/). Specimens examined: Indonesia. Java. West Java Province: Megamendung (probably), C. L.Blume s.n. (BO, K); Preanger, Cidadap, Cibeber, $1000 \mathrm{~m}$ asl, 12 June 1916, R. C. Bakhuizen v/d Brink 1617 (BO, L); Res. Batavia, G. Limoes (Boerangrang), $1200 \mathrm{~m}$ asl, 23 July 1920, R. C. Bakhuizen v/d Brink 4393 (BO); Preanger, Boschterrein Takoka, Distr. Djampang-Wetan, Afd. Tjiandjoer, $1000 \mathrm{~m}$ asl, 16 February 1894, S. H. Koorders 15093 (BO, L); Preanger Regentschappen, G. Tjikoraj, Z. O. Helling, Pasir Walang boven Nanggerang, 950-1000 m asl, 17 August 1913, C. A. Backer 8731 (BO); Preanger Regentschappen, Tjoeroeg Djanggot, Cidadap, Cibeber, $900 \mathrm{~m}$ asl, 9 September 1917, C. A. Backer 22941 (BO), Preanger, Goenoeng Boeled, Cidadap, Cibeber, $1000 \mathrm{~m}$ asl, 20 October 1923, $W$. F. Winckel 1741 (BO, L); Preanger, Tjadas Malang, Cibeber, $1000 \mathrm{~m}$ asl, 26 October 1919, W. F. Winckel 449 (BO, L); Preanger, G.
Beser, Tjidadap, Tjibeber, $1000 \mathrm{~m}$ asl, 3 July 1918, W. F. Winckel 220 (BO, L); Ga. Malang, Takoka Nat. Res., $1100 \mathrm{~m}$ asl, 15 June 1975, G. G. Hambali 722 (BO); G. Batoe, Cianten, Lewiliang, Buitenzorg, $1000 \mathrm{~m}$ asl, 31 August 1918, C. A. Backer 25819 (BO); Cigombong, Sukaraja, Sukabumi, 1983, E. A. Widjaja s.n. (BO); Mount Salak, facing east, upper Lido Village, 1000-1200 m asl, 22 February 2000, H. Wiriadinata \& W. S. Hoover 31193 (BO); Mt. Halimun Nat. Park Cikaniki, 1000-1400 m asl, 7 March 2000, H. Wiriadinata, W. S. Hoover \& J. Hunter 31339 (BO); Mount Salak, Leg. Ign. s.n. (BO); Pasir Walang, Nanggerang, 1913, C. A. Backer 8731 (BO); Batavia, Nirmala, $1100 \mathrm{~m}$ asl, 24 December 1913, C. A. Backer 11068 (BO); G. Beser, no van Gedeh, Desa Ciherang, Kampung Tunggilis, near top, 1000-1350 m asl, 11 March 1940, C. G. G. J. v. Steenis 11773 (BO, L), G. Beser, Cianjur, $1370 \mathrm{~m}$ asl, 3 September 1933, C. G. G. J. v. Steenis 5465 (BO); Mount Salak, Prope Tjoeroeg, 2 May 1895, Hallier s.n. (BO); Bogor, Kecamatan Pamijahan, Desa Purabakti, Kampung Padajaya, G. Halimun foot near Cianten tea plantation, $940 \mathrm{~m}$ asl, 25 May 2000, E. A. Widjaja 7428 (BO); Bogor, Kecamatan Pamijahan, Desa Purabakti, Kampung Padajaya, G. Halimun foot near Cianten tea plantation, $940 \mathrm{~m}$ asl, 25 May 2000, E. A. Widjaja 7430 (BO); Leuwiliang, Purwabakti Village, Garehong-Keneng, G. Halimun, $946 \mathrm{~m}$ asl, 24 April 1998, E. A. Widjaja 7157 (BO); Bogor, G. Salak, Cibitung, 18 December 1982, E. A. Widjaja 1806 (BO), G. Kempul, Cianten, 30 November 1975, E. A. Widjaja 21 (BO); Sukabumi, Lengkong 12 November 1989, E. A. Widjaja 3609 (BO); W. of Djasinga, forest-reserve Djanglope, $900 \mathrm{~m}$ asl, 20 August 1954, W. Meijer 2949 (BO); Tjiandjoer, $1000 \mathrm{~m}$ asl, 7 April 1899, S. H. Koorders 32907 (BO); Mount. Salak, Prope Tjoeroeg, Leg. Ign. s.n. (BO); Cikaniki Resort 
Jurnal Biodjati 6(2):174-189, November 2021

\section{JURNAL BIODJATI}

http://journal.uinsgd.ac.id/index.php/biodjati
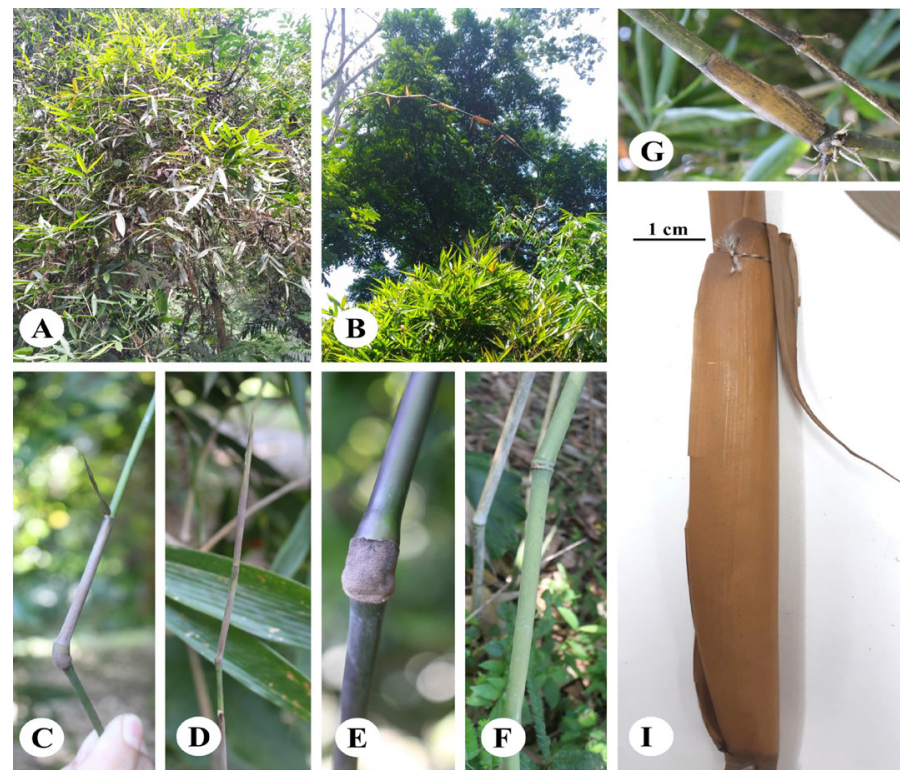

Figure 2. Dinochloa scandens: habit (A), a tip of the young culm (B), culm-sheath of the young shoot (C), a tip of the young shoot (D), young culm with purplish-green color $(\mathrm{E})$, mature culm with green color $(\mathrm{F})$, mature culm-sheath without blade due to falling early $(\mathrm{G})$, detail of culm-sheath auricles $(\mathrm{H})$, culm-sheath with a blade attached (I), detail of culmsheath ligule $(\mathrm{J})$, girdle $(\mathrm{K})$ [Photos: Syadwina H. Dalimunthe (A), I Putu Gede P. Damayanto (B, I, J, K), and Irfan Martiansyah (C-H)]
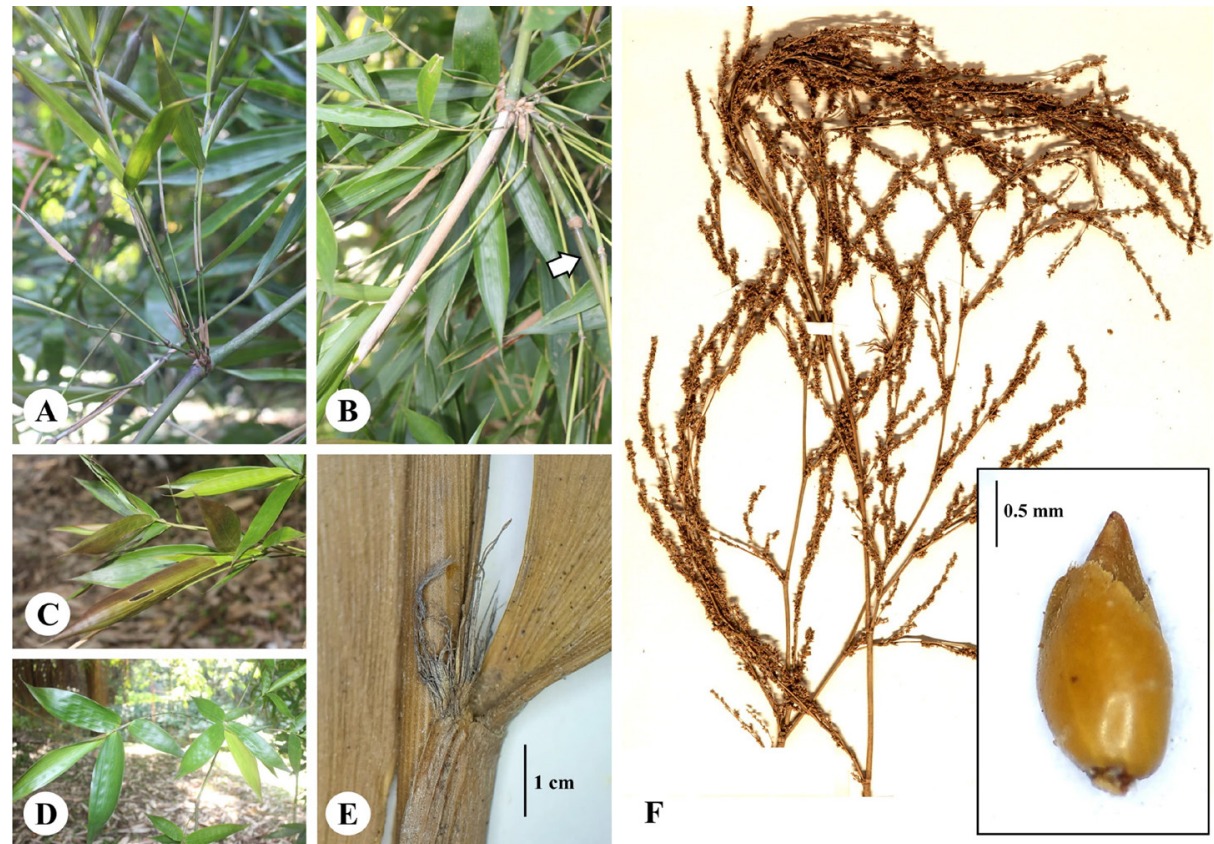

Figure 3. Dinochloa scandens: primary branch dormancy (A), primary branch developed into a full-size culm when the apex of the main culm is broken (B), young leaves with purplish-green color (C), mature leaves with green color (D), detail of leaf-sheath auricles with bristles (E), inflorescences (F) and spikelet (inset) [Photos: Irfan Martiansyah (AD), I Putu Gede P. Damayanto (E, inset F), and Bioportal, 2021 (F)] 


\section{JURNAL BIDDJATI}

http://journal.uinsgd.ac.id/index.php/biodjati

Station, 9 October 2019 S. H. Dalimunthe 20 (BO). Banten Province: Ujungkulon Nature Reserve, Gunung Pajung, $400 \mathrm{~m}$ asl, 31 December 1963, N. Wirawan s.n. (BO); Goenoeng Kantjang, Distr. Lebak Kidoel, Res. Bantam, 9 June 1912, S. H. Koorders 41410 (BO); Bantam, G. Kencana, G. Kendeng, 300 m asl, 13 June 1911, C. A. Backer 1292 (BO); Pulau Panaitan (Prinseneiland) between Tg. Manik and n. coast (w. peninsula), $20 \mathrm{~m}$ asl, 19 September 1951, J. v. B. Waalkes 468 (BO, L). Living plants examined: Bogor Botanic Gardens, Research Center for Plant Conservation and Botanic Gardens, LIPI, Bogor, 6³6'00" S, 10647'44” E, $270 \mathrm{~m}$ asl. Additional specimen examined (Dinochloa macrocarpa): the Philippines, Island of Sibuyan, Province of Capiz, Magallanes (Mt. Giting-Giting), March 1910, A. D. E. Elmer 12059 (F code V0046690F, V0046691F; K code K000290819, K000290820; NY code 380691).

\section{Distribution}

Based onherbarium specimen data(Table 1), D. scandens distributed in the western part of Java (Province of West Java and Banten), Indonesia (Figure 4). Most populations can be found around Bogor, Sukabumi, and Cianjur in West Java Province. The easternmost area where this species can be still found is around Purwakarta (Cahyanto et al., 2018) and Garut, West Java Province, while the westernmost area is Panaitan Island, Banten Province. This species has never been previously reported growing outside the mainland of the island of Java. The distribution of $D$. scandens on Panaitan Island is a new record.

Vorontsova et al. (2016), POWO, GBIF and some others (i.e. Priyadi et al., 2010; Cleary, 2016; Jamun et al., 2020; Sharief \& Panda, 2020; Sujarwanta \& Zen, 2020a, 2020 b) suggest that $D$. scandens can be found outside of Java (e.g. Sumatra, Borneo, Lesser Sunda Islands, the Andaman Islands, Nicobar Islands, and Thailand). The information of $D$. scandens in POWO and GBIF, however, is outdated. For example, the specimen of Brand 24573(K)fromLahadDatu, Malaysia(Borneo) which was used as a reference for determining distribution areas of $D$. scandens in the POWO and GBIF database is a new species, $D$. darvelana S.Dransf. (see Dransfield, 1989).

Further studies by Dransfield have also shown that some specimens of Dinochloa from Malaysia, previously identified as $D$. scandens (Dransfield, 1981), belong to $D$. robusta S.Dransf. (Dransfield, 1992) and D. malayana S.Dransf. (Dransfield, 1996). Several Dinochloa specimens from Indonesia (Central and East Java), previously suspected as $D$. scandens, have been identified later as D. matmat S.Dransf. \& Widjaja (Dransfield \& Widjaja, 2000). It appears that Priyadi et al. (2010) and Vorontsova et al. (2016) missed this information and reported D. scandens to be widely distributed. Jamun et al. (2020) stated that $D$. scandens was found in East Nusa Tenggara, Lesser Sunda Islands and is used in medicine by local communities. Unfortunately, they did not provide photographs and descriptions of the species. It could be that the species is not D. scandens, but D. kostermansiana S.Dransf., the member of Dinochloa reportedly found in East Nusa Tenggara (Dransfield, 1996; Widjaja, 2001a; Damayanto, 2016, 2017; Damayanto et al., 2018). Furthermore, Sujarwanta \& Zen (2020a, 2020b) stated that $D$. scandens was found in Lampung, Sumatra. However, the photographs presented were less clear and the description of the species was also lacking some details. We suspect that this species could be D. glabrescens Widjaja that is found in Lampung (Widjaja, 1997; Damayanto, 2018). Cleary (2016) stated that 


\section{JURNAL BIODJATI}

http://journal.uinsgd.ac.id/index.php/biodjati

D. scandens occurred in East Kalimantan (Borneo), however, there is no description or photograph available. This information needs to be evaluated since some Dinochloa species occurred there (see Dransfield, 1981, 1989).

Dinochloa scandens collected from

West Java were reportedly introduced to the Baturaden Botanic Gardens, Central Java (Sukma \& Lianah, 2019). This report, however, needs to be evaluated - the description and photograph provided by Sukma \& Lianah (2019) are insufficient to accurately identify the species. In East Java, Mudiana et al. (2020) mentioned that D. scandens was found in Alas Purwo. Unfortunately, the photographs provided were unclear and there was no species description available. It is suspected that this species is D. matmat reported occurring in Central and East Java (Dransfield \& Widjaja, 2000).

Dinochloa scandens was reported to occur in the Nicobar (Sharief \& Panda, 2020) and Andaman Islands, India (Veenakumari et al., 1997), nevertheless species description and photographs are lacking. Based on the Checklist of the Grasses of India (Kellogg et al., 2020), D. scandens was reportedly native to Indonesia and was cultivated in Andaman and Nicobar Islands. However, Kumari (2019) reported that the presence of $D$. scandens in the Indian species checklist was doubtful. The cultivation of D. scandens in Andaman and Nicobar Islands is still questionable since $D$. scandens is a wild bamboo and is considered less useful compared to Bambusa, Dendrocalamus, and Gigantochloa. Thus, the possibility of $D$. scandens being cultivated is very low. Kellogg et al. (2020) also included $D$. andamanica Kurz in their checklist. On the other hand, Naithani et al. (2000) has transferred $D$. andamanica to variety $D$. scandens var. andamanica (Kurz) Naithani. Ohrnberger(1999) mentioned that D. scandens Damayanto et al. name was misapplied to $D$. andamanica. It needs further study to clarify the existence of $D$. andamanica or D. scandens var. andamanica in Andaman and Nicobar Islands.

Here we show that D. scandens is an endemic species to the western part of Java (Banten and West Java Province) and the satellite island of Panaitan, concurrent with the previous work of Dransfield $(1994,1996)$, Dransfield \& Widjaja (1995), Damayanto (2018), and Widjaja (2019). From this study, D. scandens in West Java were found in several mountainous protected forest areas (Mount Gede-Pangrango National Park and Mount Halimun-Salak National Park). These national parks should support the continued existence of $D$. scandens in West Java. The conservation of $D$. scandens has been conducted in Indonesia. Dinochloa scandens was reportedly planted in Cibodas Botanic Gardens at area XX.A.22-22a-22b, 25, 26, 27, 28 and XX.B. 22-22a collected from West Java (Sujarwo et al., 2019) and Bogor Botanic Gardens at area XIII.K.14 collected from West Java (Ariati et al., 2019). Solikin (2004) reported D. scandens was cultivated in Purwodadi Botanic Gardens at area XII.J.I. 42 , without information on the origin of the collection. However, according to Lestarini et al. (2012) and Makoyana (2021), there were no $D$. scandens in Purwodadi Botanic Gardens cultivated and there is also no data on plant species planted in area XII.J.I. 42.

\section{Habitat Preference}

Based on notes of herbarium specimen, D. scandens is largely a forest species found on ridge slopes, humid mountains, in primary lowlands, secondary forests and near the beach. Dinochloa is rarely found in undisturbed primary rainforests. Dinochloa becomes abundant in gaps or at forest margins by roadsides when the forest is disturbed by 


\section{JURNAL BIDDJATI}

http://journal.uinsgd.ac.id/index.php/biodjati

road-constructions or logging (Dransfield, secondary forests which have frequent gaps 1996). Therefore, D. scandens is easily in the forest canopy. This species grows in found on the ridge slopes of the forest and sandy and limestone soils (Widjaja, 2001b).

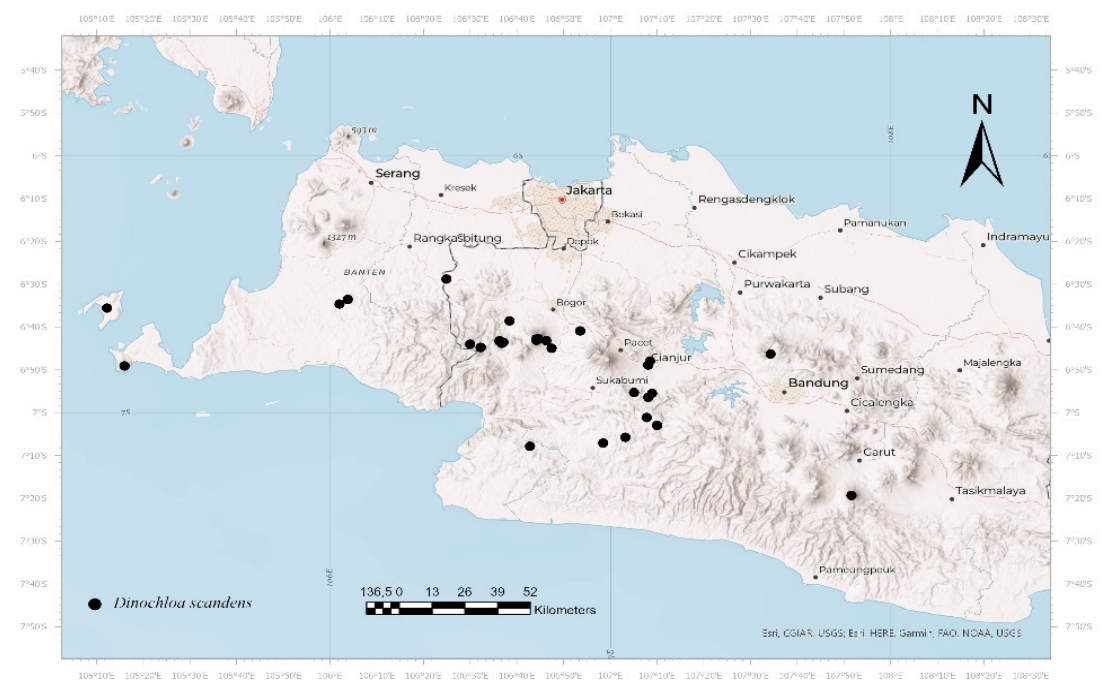

Figure 4. Distribution of Dinochloa scandens in the western part of Java and vicinity island, Indonesia

This species is found in lowland to mountain forests at $20 \mathrm{~m}$ asl (Panaitan Island, Banten Province) to $1400 \mathrm{~m}$ asl (West Java), but mostly found at $800-1200 \mathrm{~m}$ asl (Figure 5-6). Broken down into political region, $D$. scandens is distributed between $20-1000 \mathrm{~m}$ asl and 200-1400 m asl in Banten Province and West Java Province, respectively. Most plants in West Java, however, were recorded in the highlands, between 700-1000 m asl. This altitude distribution is following those of Dinochloa spp. in Sulawesi, the center of Dinochloa species diversity (Ervianti et al., 2019), where they can be found at 47-953 $\mathrm{m}$ asl (Widjaja, 2009; Ervianti et al., 2019).

Generally, D. scandens is distributed in an area with annual rainfall between 20004000 mm (Figure 7). In Banten Province, this species has been distributed in an area with 3000-4000 mm/year. In West Java Province, the species was distributed in the area with an annual rainfall of 2000-4000 mm. However, most records were found in an area with an annual rainfall of 2000-3000 mm. In Sulawesi, Dinochloa species reportedly grow in areas with an annual rainfall of 1000-3000 mm (Ervianti, 2015). Therefore, it is likely that the area with an annual rainfall of 2000-3000 mm is the most suitable habitat for $D$. scandens.

\section{Notes on Synonymy}

Vorontsova et al. (2016) include $D$. macrocarpa Elmer (see Elmer, 1915) as a synonym of $D$. scandens. The type specimen of D. macrocarpa [Elmer 12059 (F!, K!, NY!)] was collected from Magallanes (Mt. Giting-giting; the correct name is Mt. GuitingGuiting), Sibuyan Islands, Province of Capiz (now Romblon Province), the Philippines (Elmer, 1915) (Figure 8). Dransfield \& Widjaja (1995), however, suggested that the records of $D$. scandens in the Philippines, Borneo and Peninsular Malaysia were based upon incorrect identification. 
Jurnal Biodjati 6(2):174-189, November 2021

\section{JURNAL BIODJATI}

http://journal.uinsgd.ac.id/index.php/biodjati

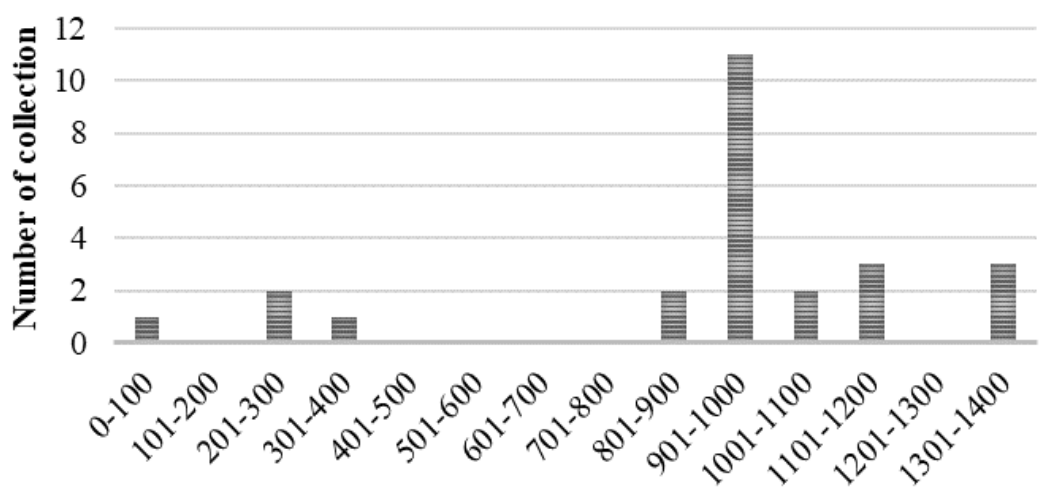

Altitude (m)

Figure 5. Altitude distribution of Dinochloa scandens based on specimen herbarium and living plant collections

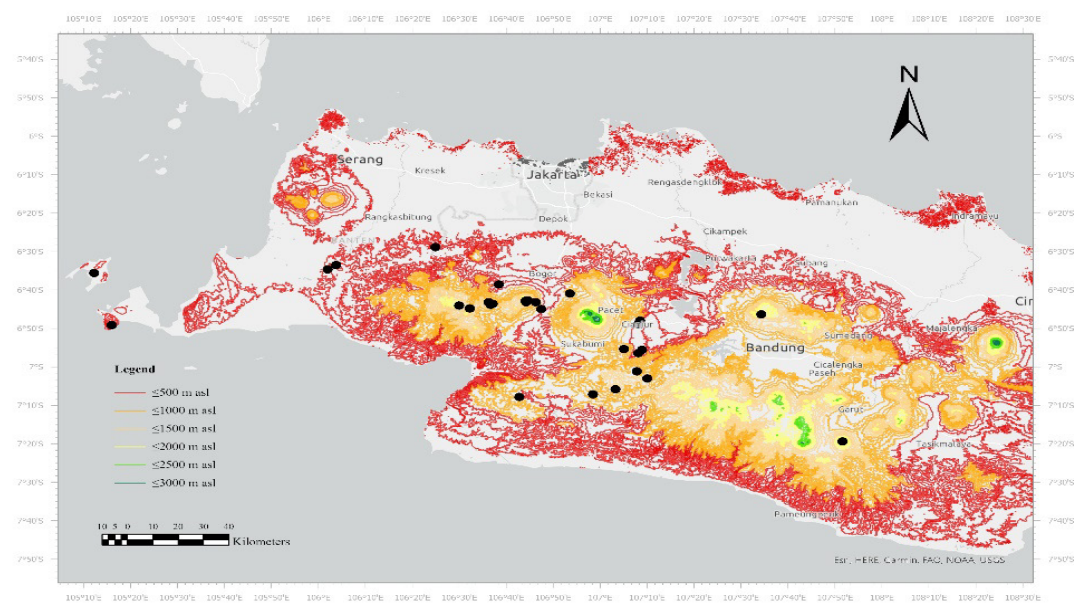

Figure 6. Overlapping maps of altitude and species distribution of Dinochloa scandens

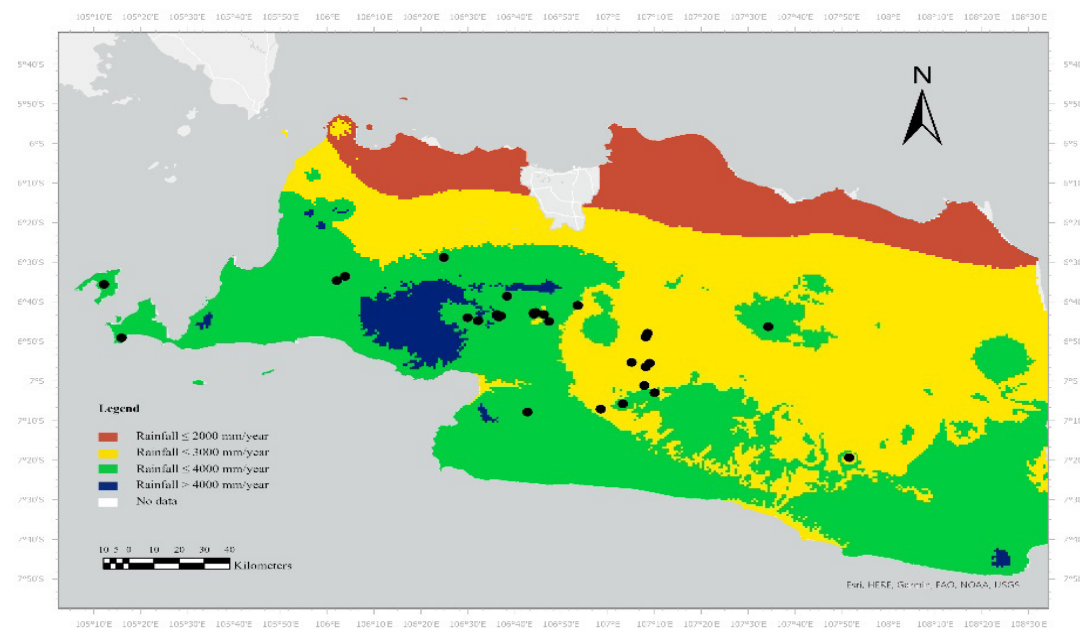

Figure 7. Overlapping maps of annual rainfall and species distribution of Dinochloa scandens 


\section{JURNAL BIDDJATI}

http://journal.uinsgd.ac.id/index.php/biodjati

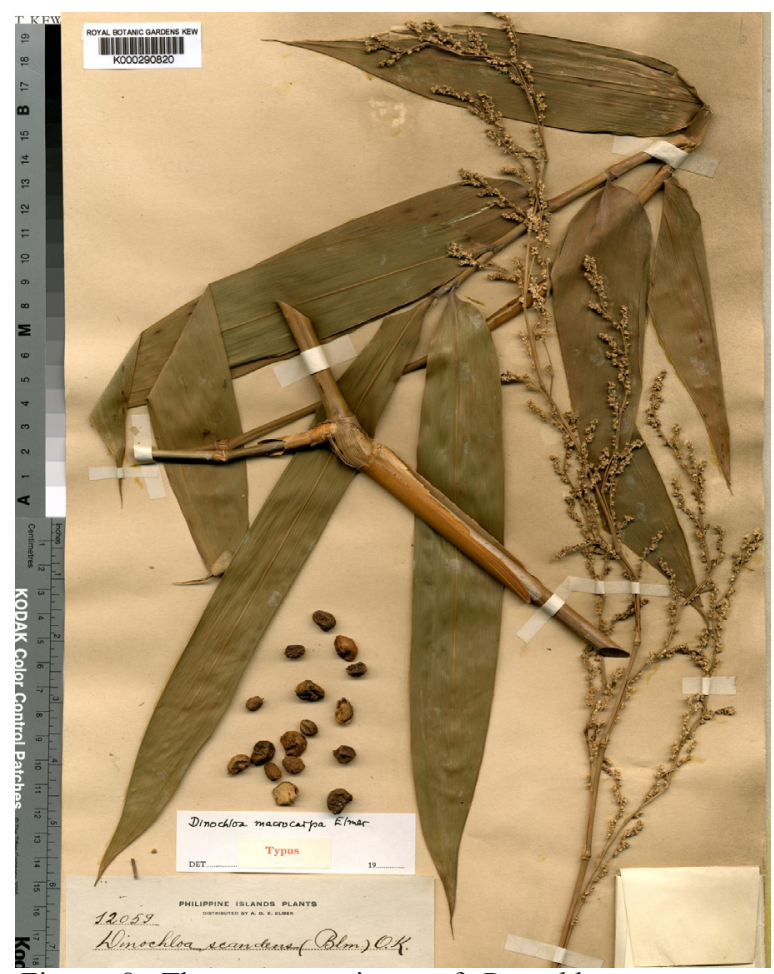

Figure 8. The type specimen of Dinochloa macrocarpa (Elmer 12059, specimen code: K000290820) stored in Herbarium of Royal Botanic Gardens, Kew (K), England (Herbweb, 2021)

The fruits of D. macrocarpa are bigger (at least $1.25 \mathrm{~cm}$ long when mature) than those of D. scandens (about $7 \mathrm{~mm}$ long). Furthermore, the fruit shape of $D$. macrocarpa is subglobose or ellipsoid, while that of $D$. scandens fruits is globose (see Elmer, 1915; Dransfield, 1996). In Dinochloa, the fruits provide the most useful diagnostic characters (Dransfield, 1981), presumably more important than the flowers (Dransfield, 1996). Hence, we suggest that D. macrocarpa is morphologically distinct from $D$. scandens and propose $D$. macrocarpa as an accepted name for a bamboo species from the Sibuyan Islands of the Philippines, concurrent with the previous work of Elmer (1915) and Ohrnberger (1999).

Dinochloa scandens is endemic to West Java and Banten Provinces, Indonesia. This bamboo can be found in lowland to mountain forests with an altitude of 20-
$1400 \mathrm{~m}$ and an annual rainfall of 3000$4000 \mathrm{~mm}$. Most populations occur between 800-1200 $\mathrm{m}$ and in areas of annual rainfall between 2000-3000 mm. We support $D$. macrocarpa as an accepted name for a bamboo species from the Philippines removing it from synonymy with $D$. scandens.

\section{ACKNOWEDGMENTS}

We thank the Head of Cikaniki Resort Station, Gunung Halimun-Salak National Park, West Java for the permission to carry out fieldwork. We thank Rani Asmarayani (Herbarium Bogoriense, Indonesia) for comments that greatly improved the manuscript. Liam A. Trethowan (Herbarium Royal Botanic Gardens, Kew, UK) is acknowledged for his assistance with written English. We thank Pushpa Kumari (Central National Herbarium, India) for the discussion and references provided. Irfan Martiansyah (Bogor Botanical Garden, Indonesia) is acknowledged for the photographs.

\section{REFERENCES}

Ariati, S. R., Astuti, R. S., Supriyatna, I., Yuswandi, A. Y. Setiawan, A., Saftaningsih, D. \& Pribadi, D. O. (2019). An Alphabetical List of Plant Species Cultivated in the Bogor Botanic Gardens. Bogor: Indonesian Institute of Sciences, Center for Plant Conservation, Bogor Botanic Gardens.

Buse, L. H. (1854). Gramineae. In: Miquel, F. A. W. Plantae Junghuhnianae: Enumeratio Plantarum, Quas, in Insulis Java et Sumatra. Leiden: LugduniBatavorum.

Cahyanto, T., Efendi, M., Shofara, R. M., Dzakiyyah, M., Nurlaela \& Satria, P. G. (2019). Short Communication: 


\section{JURNAL BIDDJATI}

http://journal.uinsgd.ac.id/index.php/biodjati

Floristic Survey of Vascular Plant in the Submontane Forest of Mt. Burangrang Nature Reserve, West Java, Indonesia. Biodiversitas, 20(8), 2197-2205.

Clark, L. G. (1986). Systematics of Chusquea Section Chusquea, Section Swallenochloa, Section Verticillatae, and Section Serpentes (Poaceae: Bambusoideae). Theses. Iowa: Graduate Programs, Iowa State University.

Cleary, D. F. R. (2016). Diversity and Composition of Plants, Butterflies and Odonates in an Imperata cylindrica Grassland Landscape in East Kalimantan, Indonesia. Journal of Tropical Ecology, 32, 555-560.

Damayanto, I. P. G. P. (2016). Lulu Ura Bambu Liar Merambat Endemik Nusa Tenggara Timur. Buletin Kakatua, 1, 14-16.

Bioportal (2021). Dinochloa scandens (Blume) Kuntze, Registration Number L.1247151. Retrieved from https:// medialib.naturalis.nl/file/id/L.1247151/ format/master.

Damayanto, I. P. G. P. (2017). Climbing Bamboos of Lesser Sunda Islands, Indonesia. Proceedings The 1st SATREPS Conference 1. Indonesian Institute of Sciences, Center for Plant Conservation, Bogor Botanic Gardens. Bogor, 30 ${ }^{\text {th }}$ November 2017. pp. 90-93.

Damayanto, I. P. G. P. (2018). Dinochloa malayana S.Dransf. (Poaceae: Bambusoideae), a New Record for Indonesia. Reinwardtia, 17(1), 35-37.

Damayanto, I. P. G. P., Mahendra, T. \& Rosalina, D. (2018). Bamboo Diversity at Laiwangi-Wanggameti National Park, Sumba, Indonesia. Buletin Kebun Raya, 21(1), 45-52.

Djarwaningsih T., Sunarti, S. \& Kramadibrata, K. (2002). Panduan Pengolahan dan
Pengelolaan Material Herbarium serta Pegendalian Hama Terpadu di Herbarium Bogoriense. Bogor: Herbarium Bogoriense, Bidang Botani, Pusat Penelitian Biologi-LIPI.

Dransfield, S. (1981). The Genus Dinochloa (Gramineae-Bambusoideae) in Sabah. Kew Bulletin, 36(3), 613-633.

Dransfield, S. (1989). A New Species of Dinochloa (Gramineae-Bambusoideae) from Borneo. Kew Bulletin, 44(3), 435437.

Dransfield, S. (1992). Dinochloa robusta, a New Species of Bamboo (Gramineae: Bambusoideae) from Sabah and Palawan. Kew Bulletin, 47(3), 402.

Dransfield, S. (1994). Bamboo Resources in Thailand: How Much Do We Know?. Proceedings 4th International Bamboo Workshop. Ottawa, (no date information), IDRCFAO-UNDP. pp. $1-4$.

Dransfield, S. (1996). New Species of Dinochloa (Gramineae-Bambusoideae) in Malesia and Notes on the Genus. Kew Bulletin, 51(1), 103-117.

Dransfield, S. \& Widjaja, E. A. (1995). Plant Resources of South-East Asia No. 7 Bamboos. Leiden: Backhuys Publishers.

Dransfield, S. \& Widjaja, E. A. (2000). Dinochloa matmat, a New Bamboo Species (Poaceae-Bambusoideae) from Java, Indonesia. Kew Bulletin, 55(2), 495-497.

Elmer, A. D. E. (1915). Two Hundred Twenty Six New Species-I. Leaflets of Philippine Botany, 7(114), 2543-2700.

Ervianti, D. (2015). Keanekaragaman dan Sebaran Spasial Jenis Bambu di Sulawesi. Theses. Jakarta: Universitas Negeri Jakarta.

Ervianti, D., Widjaja, E. A. \& Sedayu, A. (2019). New Species of Climbing and 


\section{JURNAL BIDDJATI}

http://journal.uinsgd.ac.id/index.php/biodjati

\begin{abstract}
Scrambling Bamboo from Sulawesi, Indonesia. Reinwardtia, 18(2), 115-132.

Girmansyah, D., Santika, Y. \& Suratman. (2006). Index Herbariorum Indonesianum. Bogor: Pusat Penelitian Biologi, LIPI.

Girmansyah, D., Santika, Y., Rugayah \& Rahajoe, J. S. (2018). Index Herbariorum Indonesianum. Jakarta: LIPI Press.
\end{abstract}

Herbweb. (2021). Dinochloa macrocarpa Elmer, K000290820. Retrieved from http://specimens.kew.org/herbarium/ K000290820.

Holmgren, P. K., Keuken, W. \& Schofield, E. K. (1981). Index Herbariorum, the Guide to the Location and Contents of the World's Public Herbaria, Part I: the Herbaria of the World. Seventh edition. Utrecht: Bohn, Scheltema \& Holkema.

Jamun, R., Hendra, M. \& Hariani, N. (2020). Keanekaragaman Tumbuhan Obat di Suku Manggarai Kecamatan Ndoso Kabupaten Manggarai Barat Nusa Tenggara Timur (NTT). Jurnal Pendidikan Matematika dan IPA, 1(2), 285-299.

Kamitani, S., Kahono, S., Ubaidillah, R., Herwint, S. \& Partomihardjo, T. (2004). A New Species of the Genus Cofana (Hemiptera: Auchenorrhyncha: Cicadellidae) Associated with a Climbing Vine of Bamboo in Java, Indonesia. Esakia, 44, 135-141.

Kellogg, E. A., Abbott, J. R., Bawa, K. S., Gandhi, K. N., Kailash, B. R., Ganeshaiah, K. N., Shrestha, U. B. \& Raven, P. (2020). Checklist of the Grasses of India. PhytoKeys, 163, $1-560$.

Kumari, P. (2019). Bambusoideae in India: an Updated Enumeration. Plantae Scientia, 1(6), 99-117.

Kuntze, O. (1981) Revisio Generum
Plantarum: Vascularium Omnium Atque Cellularium Multarum Secundum Leges Nomeclaturae Internationales Cum Enumeratione Plantarum Exoticarum in Itinere Mundi Collectarum Pars II. Leipzig: Arthur Felix; London: Dulau \& Co.; New York: Gust. E. Steehert; Paris: Charles Klinecsieck; Milan: U. Hopeli.

Lestarini, W., Matrani, Sulasmi, Trimanto, Fauziah \& Fiqa, A. P. (2012). An Alphabetical List of Plant Species Cultivated in Purwodadi Botanic Garden. Purwodadi: Purwodadi Botanic Garden, Indonesian Institute of Sciences.

Makoyana (Manajemen Koleksi Kebun Raya Indonesia). (2021). Manajemen Koleksi Kebun Raya Indonesia. Retrieved from http://makoyana.lipi.go.id.

McClure, F. A. (1945). Suggestions on How to Collect Bamboos. Washington DC: Division of Latin American Agriculture, Office of Foreign Agricultural Relations. Mudiana, D., Renjana, E., Firdiana, E. R. Ningrum, L. W., Angio, M. H. \& Irawanto, R. (2020). Penambahan koleksi Tumbuhan Kebun Raya Purwodadi Melalui Sksplorasi di Taman Nasional Alas Purwo. Jurnal Penelitian Kehutanan Wallacea, 9(2), 83-92.

Munro, W. (1868). A Monograph of the Bambusaceae, Including Description of All the Species. Transactions of the Linnean Society of London, 26(1), 1-157.

Naithani, H., Chandra, S., Rajesh \& Pal, M. (2000). Nomenclature, Distribution and Phenology of Bamboos Dinochloa and Gigantochloa in Andaman \& Nicobar Islands. Indian Forester, 126(9), 10081012.

Ohrnberger, D. (1999). The Bamboos of the World: Annotated Nomenclature and Literature of the Species and the Higher 


\section{JURNAL BIDDJATI}

http://journal.uinsgd.ac.id/index.php/biodjati

\begin{tabular}{l}
\hline and Lower Taxa. Amsterdam: Elsevier. \\
Partasasmita, R., An'amillah, A., Iskandar, J., \\
Mutaqin, A. Z., Annisa \& Ratnangsih, \\
N. (2017). Traditional Knowledge of \\
Bamboo and Its Role in Karangwangi \\
Village Cianjur, West Java: Implications \\
for Management of Cultural Keystone \\
Species. Biodiversitas, 18(1), 275-282.
\end{tabular}

Priyadi, H., Takao, G., Rahmawati, I., Supriyanto, B., Nursal, W. I. \& Rahman, I. (2010). Five Hundred Plant Species in Gunung Halimun Salak National Park, West Java, a Checklist Including Sundanese Names, Distribution and Use. Bogor: Cifor.

Setiawati, T., Mutaqin, A. Z., Irawan, B., An'amillah, A. \& Iskandar, J. (2017). Species Diversity and Utilization of Bamboo to Support Life's the Community of Karangwangi Village, Cidaun Sub-District of Cianjur, Indonesia. Biodiversitas, 18(1), 58-64.

Sharief, M. U. \& Panda, S. P. (2020). Folklore Artefacts of Aboriginal Shompen Tribe in Great Nicobar Island, India. Retrieved from https://doi.org/10.1007/ s10722-020-01027-x.

Solikin. 2004. Jenis-jenis Tumbuhan Suku Poaceae di Kebun Raya Purwodadi. Biodiversitas, 5(1), 23-27.

Steenis-Kruseman, M. J. V. (1950). Malaysian Plant Collectors and Collections. Flora Malesiana, 1(1), 1-639.

Sujarwanta, A. \& Zen, S. (2020a). Identifikasi Jenis dan Potensi Bambu (Bambusa sp.) sebagai Senyawa Antimalarial. Bioedukasi, 11(2), 131-15.

Sujarwanta, A. \& Zen, S. (2020b). Etnobotani

$\begin{array}{lcr}\text { Tanaman } & \text { Bambu di } & \text { Kecamatan } \\ \text { Semaka } & \text { Kabupaten } & \text { Tanggamus } \\ \text { Provinsi } & \text { Lampung. } & \text { Retrieved } \\ \text { from } & \text { https://repository.ummetro. } \\ \text { ac.id/file s/artikel/8 df2 } 85 \text { eb } 1 \text { c - } \\ \text { 39736c4753aa096facc.pdf. }\end{array}$

Veenakumari, K., Mohanraj, P. \& Sreekumar, P. V. (1997). Host Plant Utilization by Butterfly Larvae in the Andaman and Nicobar Islands (Indian Ocean). Journal of Insect Conservation, 1, 235-246.

Vorontsova, M., Clark, L. G., Dransfield, J., Govaerts, R. \& Baker, W. J. (2016). World Checklist of Bamboo and Rattans. Inbar Technical Report, 37, 1-454.

Widjaja, E. A. (1997). New Taxa in Indonesian Bamboos. Reinwardtia, 11(2), 57-152.

Widjaja, E. A. (2001a). Identikit Jenisjenis Bambu di Kepulauan Sunda Kecil. Bogor: Herbarium Bogoriense, Balitbang Botani, Puslitbang BiologiLIPI.

Widjaja, E. A. (2001b). Identikit Jenis-jenis Bambu di Jawa. Bogor: Pusat Penelitian Biologi-LIPI.

Widjaja, E. A., Astuti, I. P. \& Arinasa, I. B. K. (2004). New Species of Bamboos (Poaceae-Bambusoideae) from Bali. Reinwardtia, 12(2), 199-204.

Widjaja, E. A. (2009). Three New Species of Dinochloa (Poaceae, Bambusoideae) with Erect Culm Sheath Blades from Sulawesi, Indonesia. Reinwardtia, 12(5), 435-440.

Widjaja, E. A. (2019). The Spectacular Indonesian Bamboos. Jakarta: Polagrade. 